\title{
ALINHAMENTO DE FERRAMENTAS DE GESTÃO DO CONHECIMENTO EM UMA INSTITUIÇÃO DE ENSINO SUPERIOR
}

http://dx.doi.org/10.5902/2318133837497

\author{
Simone Boruck Klein ${ }^{1}$ \\ Salette Silveira Azevedo \\ Paula Bitar Silva ${ }^{3}$
}

\begin{abstract}
Resumo
O objetivo desta pesquisa foi a elaboração de um projeto de gestão do conhecimento em uma instituição de ensino superior. Buscou-se diagnosticar a realidade da gestão do conhecimento na IES pela aplicação do instrumento de Bukowitz e Williams (2002), bem como relacionar as ferramentas de gestão do conhecimento às características culturais da instituição. Trata-se de uma pesquisa intervencionista e quantitativa. Como resultado destaca-se os menores índices nos critérios de avaliação de novas formas de conhecimento e no descarte de conhecimento. A proposição foi de um projeto de gestão do conhecimento, composto de ferramentas tecnológicas wikis, blogs e portais - e não tecnológicas - requisitos de capacidade futura, quadro de ação estratégica, métricas de mudança cultural, gestão de risco e seleção de processo.

Palavras-chave: gestão do conhecimento; ferramentas de gestão do conhecimento; instituição de ensino superior.
\end{abstract}

\section{KNOWLEDGE MANAGEMENT TOOLS ALIGNMENT IN A HIGHER EDUCATION INSTITUTION}

\begin{abstract}
The objective of this research was the elaboration of a project of knowledge management in a Higher Education Institution. It was sought to diagnose the reality of knowledge management in the $\mathrm{HEl}$ by applying the instrument of Bukowitz and Williams (2002), as well as to relate knowledge management tools to the institutional characteristics of the institution. It is an interventionist and quantitative research. As a result, the lowest indexes in the evaluation criteria of new forms of knowledge and in the discard of knowledge are highlighted. The proposal was a project of knowledge management, composed of technological tools (wikis, blogs and portals) and nontechnological (requirements of future capacity, framework of strategic action, metrics of cultural change, risk management and process selection).
\end{abstract}

Key-words: knowledge management; knowledge management tools; higher education institution.

\footnotetext{
${ }^{1}$ Fundação Pedro Leopoldo, Brasil. E-mail: simoneboruck@uol.com.br.

2 Fundação Pedro Leopoldo, Brasil. E-mail: salettesilveiraazevedo@gmail.com.

${ }^{3}$ Fundação Pedro Leopoldo, Brasil. E-mail: paulabitar@yahoo.com.br. 


\section{Introdução}

ntigas formas de desenvolvimento econômico e social como a terra, o trabalho e o capital estão sendo substituídos por um ativo fundamental à sobrevivência organizacional: o conhecimento.

Com base nesse pressuposto, e em conformidade com Davenport e Prusak (1998), verifica-se que o conhecimento se tornou passível de ser criado, desenvolvido, mensurado e armazenado. Sendo assim, de acordo com Nonaka e Takeuchi (2004), constitui-se um poderoso recurso competitivo, uma vez que a explicitação do mesmo pode fornecer vantagem competitiva às organizações a um longo prazo, bem como criar conhecimento organizacional.

Para as instituições de ensino superior - IES -, a abordagem de gestão do conhecimento equivale à de outras organizações empresariais. Da mesma forma, por se tratar de uma organização produtora do próprio conhecimento, a estruturação de práticas, ferramentas e modelos de gestão têm se tornado objeto freqüente de estudos acadêmicos.

Entretanto, percebe-se o quanto é necessário criar um ambiente propício para o estabelecimento de um projeto de gestão do conhecimento, assim como para a escolha adequada das ferramentas implantadas em cada instituição. Em virtude disso, buscou-se contribuir com essa lacuna, relacionando o ambiente atual de uma IES, no que se refere à gestão do conhecimento, com as ferramentas adequadas para geri-la por meio da proposição de um projeto de gestão do conhecimento.

A problemática que norteou a pesquisa foi expressa nos seguintes termos: como alinhar um projeto de gestão do conhecimento com as características ambientais em uma IES?

Sob tal perspectiva, o objetivo geral foi a proposição de um projeto de gestão do conhecimento a partir das características ambientais da IES estudada. Os objetivos específicos foram diagnosticar a atual realidade da gestão do conhecimento na IES, fazendo uso das sete seções propostas por Bukowitz e Williams (2002) e elencar as ferramentas de gestão do conhecimento mais abrangentes na literatura acadêmica atual, inter-relacionando as características mais frágeis elencadas, após o diagnóstico da IES, com as soluções propostas pelas ferramentas de gestão do conhecimento.

Nesse sentido, pretende-se, neste artigo, apresentar uma contribuição às novas evidências empíricas, tanto para a área de Ciências Sociais Aplicadas, como também, às implicações práticas às IES que desejam implantar uma gestão do conhecimento de forma estruturada e funcional.

\section{Gestão de conhecimento e capital intelectual}

Ao tratar da gestão do conhecimento e do capital intelectual é basilar partir de seus conceitos, modelos e ferramentas disponíveis na literatura. As ferramentas elencadas da literatura internacional dividem-se, de acordo com seu escopo, em tecnológicas e não tecnológicas e apresentam características relacionadas ao objetivo final de cada uma delas. 
Alavancar o conhecimento nas organizações é considerado, na contemporaneidade, um caminho para a vantagem competitiva e à sustentabilidade das organizações. Sendo assim, o desempenho organizacional está relacionado às questões de conhecimento e à aprendizagem organizacional.

A palavra conhecimento, oriunda do latim cognoscere E significa, de maneira ampla, conhecer pelos sentidos. Sob essa ótica sua conceituação apresenta características distintas devido à sua intangibilidade. Segundo Davenport (1998), conhecimento é, pois, uma mistura de experiências condensadas que apresentam valores, informações contextuais e insights experimentados, os quais se fundem em uma só estrutura. Figueiredo (2005, p. 43) afirma que conhecimento "é o ato ou efeito de conhecer. Informação, notícia ou ciência. Prática da vida. Experiências. Discernimento, critério, apreciação, instrução, saber, erudição."

Nessa perspectiva, é possível identificar diferenças entre dados, informações, conhecimento e sabedoria. Não obstante, para que ocorra a gestão do conhecimento, há necessidade de uso de todas essas dimensões.

Importante ressaltar que existem duas abordagens acadêmicas relacionadas ao conhecimento organizacional, isto é, as literaturas referentes ao capital intelectual e à abordagem de gestão do conhecimento. Em sua gênese, ambas são integradas, porém, no decorrer das décadas, rompem-se e formam diferentes linhas de pesquisa.

No quadro 1 são apresentados alguns estudos que apontam o desenvolvimento dessas linhas de pesquisa e seus principais autores entre as décadas de 1980 e 1990. A partir de 2000 a opção passou a ser a de manter somente os estudos relacionados à gestão do conhecimento, em virtude da distinção de foco e abrangências das respectivas linhas de pesquisa.

Quadro 1 -

Evolução da literatura científica relacionada à gestão do conhecimento e ao capital Intelectual

\begin{tabular}{|c|c|c|}
\hline Época & Autor & Foco e termos usados \\
\hline \multirow[t]{2}{*}{ Déc.de 1980} & Porter & Conhecimento estratégico \\
\hline & Nonaka e Takeuchi & Compartilhamento de conhecimentos \\
\hline \multirow[t]{10}{*}{ Déc. de 1990} & Choo & Organização do conhecimento \\
\hline & Davenport e Prusak & Prática do Conhecimento \\
\hline & Drucker & Sociedade do conhecimento \\
\hline & Edvinsson e Malone & $\begin{array}{l}\text { Capital humano, estrutural, cliente e capital } \\
\text { organizacional }\end{array}$ \\
\hline & Quinn & Inteligência Organizacional \\
\hline & Senger & $5^{\text {a }}$ disciplina: pensamento sistêmico \\
\hline & Stewart & Capital humano, capital estrutural e capital cliente \\
\hline & Kaplan-Norton & $\begin{array}{l}\text { Balanced Scorecard- Capital humano, capital } \\
\text { informacional, capital organizacional e capital } \\
\text { estrutural }\end{array}$ \\
\hline & Edvinsson & $\begin{array}{l}\text { Skandia Navigator- C. humano, c. relacional e } \\
\text { capital estrutural }\end{array}$ \\
\hline & Roos e Roos & Índice Cl- C.humano, relacional e do cliente e capital \\
\hline Regae: Rev. Gest. & Santa Maria & Pub. contínua 2019 \\
\hline
\end{tabular}




\begin{tabular}{l|l|l}
\cline { 2 - 3 } & Organizacional \\
\cline { 2 - 3 } 2000 em diante & Sveiby & Ativos intangíveis \\
\cline { 2 - 3 } & Bontis & $\begin{array}{l}\text { Peso Patente- C..humano, C. relacional e c. } \\
\text { estrutural }\end{array}$ \\
\hline & Nonaka & $\begin{array}{l}\text { Conhecimento e inovação } \\
\text { Consolidação da teoria do conhecimento } \\
\text { organizacional }\end{array}$ \\
\cline { 2 - 3 } & Terra & $\begin{array}{l}\text { Práticas gerenciais no processo de gestão do } \\
\text { conhecimento }\end{array}$ \\
\cline { 2 - 3 } & Stollenwerk & Modelo genérico de gestão do conhecimento \\
\cline { 2 - 3 } & Bukowitz e Willians & Diagnóstico de gestão do conhecimento \\
\cline { 2 - 3 } & $\begin{array}{l}\text { Probst, Raub, } \\
\text { Romhardt }\end{array}$ & $\begin{array}{l}\text { Aplicação de gestão do conhecimento com base na } \\
\text { abordagem de elementos construtivos }\end{array}$ \\
\hline
\end{tabular}

Fonte: Adaptada de Krücken-Pereira, Costa e Bolzan (2002); Ferenhof, Bialecki e Selig (2014).

De forma sintética, a literatura de capital intelectual examina os tipos de recursos intangíveis, sua mensuração e relações com os desempenhos e estratégias das organizações. Já a literatura de gestão do conhecimento aborda os mecanismos, pelos quais esses recursos podem ser gerenciados. Isso porque, por um lado, a natureza do conhecimento é discutida como um recurso, por outro, como um processo.

De acordo com Edvinsson e Malone (1997), nos estudos de capital intelectual, os ativos intangíveis contribuem para a criação de valor. Distintamente, segundo Kianto, Ritala, Spender e Vanhala (2014), os estudos de gestão do conhecimento contribuem para processos de geração, armazenamento e compartilhamento de conhecimentos, ou seja, atividades conduzidas para gerenciar o fluxo.

A gestão do conhecimento, no âmbito de uma organização, corresponde à identificação do que já se sabe, do que é possível captar, assim como organizar esse conhecimento, tendo em vista a aplicação do mesmo para geração de desempenhos superiores à organização (Stewart, 2002).

Em conformidade com os estudos de Curado e Bontis (2011), esse processo é dinâmico, intermitente e atinge a organização como um todo, uma vez que a "gestão do conhecimento é uma disciplina organizacional que une demanda e oferta de informações em apoio a processos de aprendizagem dentro das organizações, levando a um melhor desempenho" p. 1440)

Na mesma linha de raciocínio, Terra (2000) enfatiza que as redes de conhecimento são formadas interna e externamente. A princípio, de maneira informal e auto-organizada e, quando geridas pela organização, podem contribuir para seu respectivo desempenho.

\section{Modelos de gestão do conhecimento}

Diversos modelos teóricos de gestão do conhecimento foram desenvolvidos nas últimas décadas, cada um deles constituído por diferentes abordagens e dimensões.

Os modelos propostos por Nonaka e Takeuchi (1995; 2004); Terra, (2000) e Bukowitz e Williams (2002) apresentam abordagens distintas e são amplamente aceitos e validados, servindo de exemplos do quanto abrangentes podem ser as dimensões da gestão do conhecimento em organizações. 
O modelo denominado SECl, proposto por Nonaka e Takeuchi (1995), foca a criação do conhecimento e a conversão deste no plano tácito para o explícito. Este modelo é espiral, dinâmico e contínuo e contempla duas dimensões:

a) epistemológica: que combina práticas de combinação (explícito para explícito), internalização (explícito para tácito), socialização (tácito para tácito) e externalização (tácito para explícito);

b) ontológica: que transfere o conhecimento individual para o grupo, para uma organização e, posteriormente, para outras organizações.

O modelo proposto por Terra (2000) é desenvolvido com uma visão abrangente e se baseia em sete dimensões, a saber:

a) visão estratégica da alta administração;

b) cultura organizacional;

c) estrutura organizacional;

d) políticas de recursos humanos;

e) sistemas de informação;

f) mensuração de resultados;

g) aprendizado com o ambiente.

Nesse modelo o conhecimento é gerado, difundido, apropriado e aplicado por pessoas, empresas ou demais organizações. Por essa razão, há necessidade de uma coordenação sistêmica à organização, do mesmo modo que à forma individual.

No terceiro modelo, proposto por Bukowitz e Williams (2002), a divisão ocorre por processos táticos e estratégicos da gestão do conhecimento. Das sete seções quatro correspondem ao processo tático - obtenha, utilize, aprenda e contribua -, e as outras três ao processo estratégico - avalie, construa/mantenha e descarte.

$\mathrm{Na}$ figura 1 apresenta-se este modelo com as duas dimensões e as sete seções.

Figura 1-

Modelo de Bukowitz e Williams (2002).

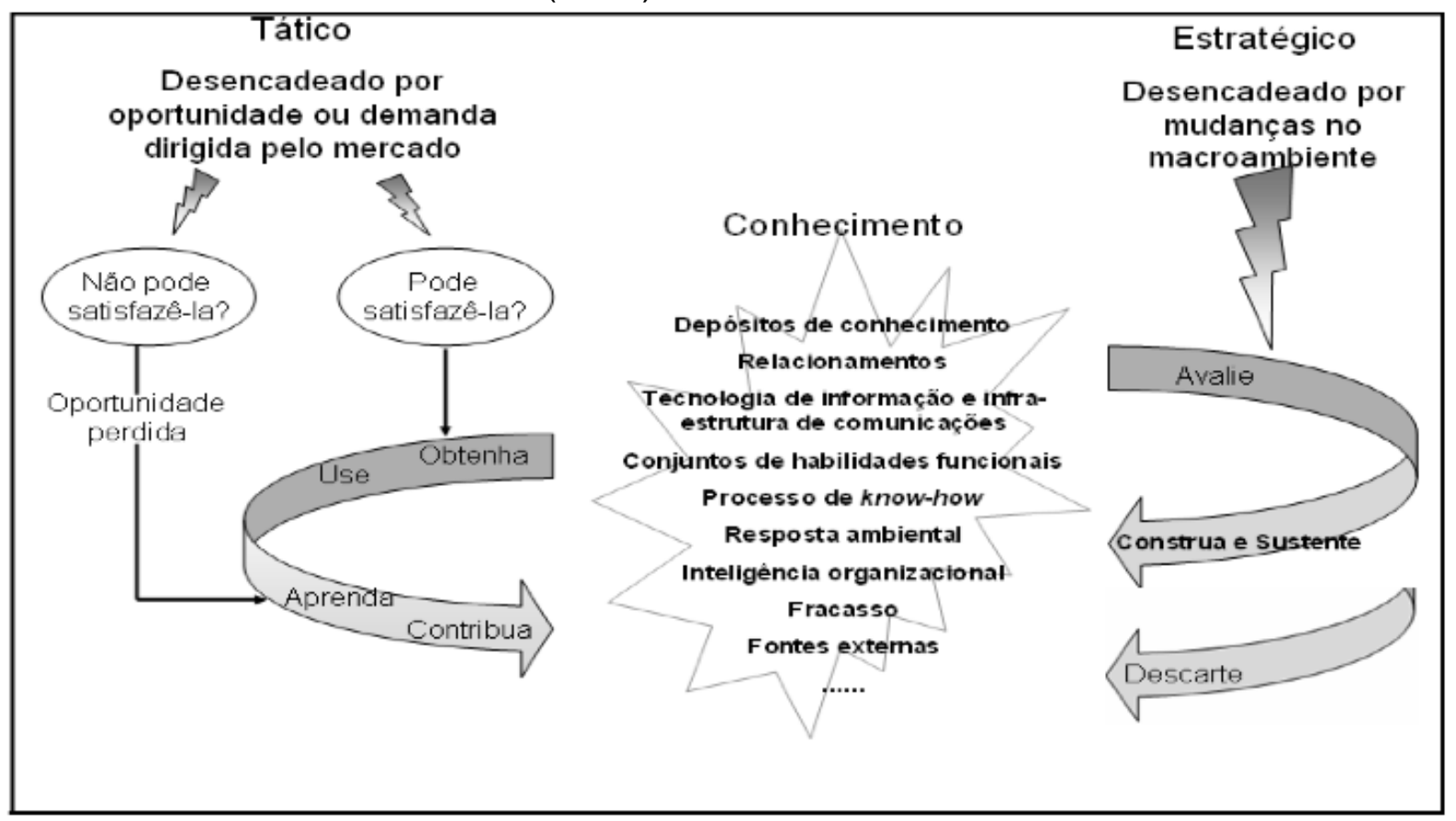

Fonte: Bukowitz e Williams (2002) 
As autoras desse modelo visam a utilizar o conhecimento para criar processos mais eficientes e efetivos e dividem a organização em ambientes internos e externos.

O processo tático foca o ambiente interno, subordinando-se às alterações de oportunidade e às demandas do mercado. Para tanto utiliza o conhecimento relativo à máxima "para gerar valor, aprender com o que se criou e, finalmente, devolver esse conhecimento para o sistema para que outros o utilizem quando abraçarem os seus próprios problemas." (Bukowitz; Williams, 2002, p. 24).

Já o processo estratégico tem seu foco no ambiente externo e na gestão do conhecimento a nível institucional para alinhar a estratégia da gestão do conhecimento à estratégia do negócio. Dessa forma, o ciclo do processo de gestão do conhecimento deve iniciar pelo ambiente interno e seguir a seguinte ordem das seções: obtenha a informação; utilize a informação; aprenda a usar o conhecimento; contribua e compartilhe o conhecimento; avalie; construa/mantenha e descarte.

Nesse sentido, ao ser formada uma base de conhecimento comum, implantada pelo compartilhamento do conhecimento, o próximo passo equivale a dirigir a atenção ao ambiente externo. Nesse foco estratégico da gestão do conhecimento o primeiro passo é avaliar o conhecimento para verificar se este pode ser utilizado e sustentado ou descartado, conforme argumenta Caldas (2009). Logo, este modelo faz uso de um diagnóstico que engloba as sete seções, realizado, neste estudo, por um questionário, melhor explicado na metodologia.

Os estudos de gestão do conhecimento se desenvolvem na Visão Baseada no Conhecimento (knowledge-based view - KBV) que aponta como pressuposto ser a criação e aplicação do conhecimento o principal fundamento da organização (Demsetz, 1991, Grant, 1996, Spender, 1996). Essa teoria, em pleno desenvolvimento, fundamentase em outra teoria, que tem como alicerce os recursos de Barney (1986) a visão baseada em recursos. Nestas teorias, o ambiente interno constitui fonte propiciadora de vantagem competitiva. Assim, o ambiente favorece a cultura do conhecimento e do capital intelectual, configurando um fator determinante às organizações para a implementação de projetos de gestão do conhecimento.

Um estudo realizado por Donate e Guadamillas (2011), ao verificar a relação entre gestão do conhecimento e a cultura organizacional, a liderança e as práticas de recursos humanos, apresenta, em seus resultados, o apontamento de que essas variáveis moderam o efeito das práticas de gestão do conhecimento. Consequentemente, o referido estudo comprova que as condições organizacionais são essenciais para uma visão baseada em conhecimento e em inovação.

A influência da cultura organizacional nas práticas de gestão do conhecimento também é enfatizada em estudos realizados por Alavi, Kayworth e Leidner (2005), tendo em vista a investigação dessa influência na gestão do conhecimento. Este estudo mostra que valores intrínsecos à cultura das organizações são também aplicados às ferramentas de gestão do conhecimento, podendo ser valores positivos ou negativos. Ou melhor, se uma organização já possui valores como colaboração, inovação e autonomia, as ferramentas de gestão do conhecimento também podem apresentar essas mesmas características, utilizadas para as devidas finalidades. 


\section{Ferramentas da gestão do conhecimento}

Os processos estruturados de gestão do conhecimento ocorrem por intermédio de projetos que utilizam sistemas de gerenciamento de conhecimento, com escopo tecnológico e ferramentas de gestão do conhecimento não tecnológicas.

Consoante Rathi e Given (2017), as ferramentas tecnológicas compõem-se da tecnologia de informação e comunicação, que atuam como facilitadoras da gestão do conhecimento. Dessa forma, a tecnologia ocupa um lugar crucial nesse grupo de ferramentas, com a captura, o compartilhamento e a organização do conhecimento. Como exemplo, podem ser citados os bancos de dados e os gerenciamentos de documentos, que são ferramentas tecnológicas com aplicações variadas e servem de base para o sistema de gestão do conhecimento tecnológico, cuja função é a de organizar, armazenar e recuperar informações. Apesar da abrangência e amplitude, outras ferramentas tecnológicas podem ser mais úteis à geração e à distribuição do conhecimento.

Já as ferramentas com escopo não tecnológico, segundo Wenger, McDermott e Snyder (2002), também são divididas em grupos distintos de utilidade. Alguns estudos mostram que o conhecimento tácito é transferido com maior eficiência nas comunicações e nas relações humanas, já que o conhecimento se dá nas relações sociais.

Não obstante, para uma prática eficaz de ferramentas de gestão do conhecimento, são necessários alinhamentos que encorajem o seu uso e que, propositadamente, baseiem-se na riqueza cultural da empresa. Além disso, para Alavi, Kayworth e Leidner (2005), são necessários sistemas com recursos e funcionalidades adaptados às culturas, nas quais eles serão utilizados, com ambientes apropriados que facilitem comportamentos e desenvolvimento de líderes para comunidades que fomentem a colaboração.

\section{Classificação das ferramentas de gestão do conhecimento}

As ferramentas e práticas de gestão do conhecimento são divididas, como mencionado, em dois grupos: tecnológicas e não tecnológicas.

No quadro 2 são apresentadas as ferramentas com escopo não tecnológico e algumas características intrínsecas às mesmas. Essas características dividem esse grupo em duas partes: gestão de recursos de conhecimento e gerenciamento do conhecimento.

Quadro 2 -

Ferramentas e práticas para gestão do conhecimento.

\begin{tabular}{|c|c|c|c|c|}
\hline & Objetivo & Ferramentas & Autores & $\begin{array}{l}\text { Nível } \\
\text { organizacional }\end{array}$ \\
\hline \multirow{8}{*}{ 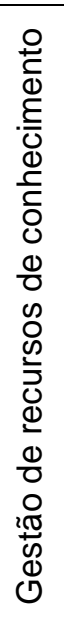 } & \multirow{3}{*}{ 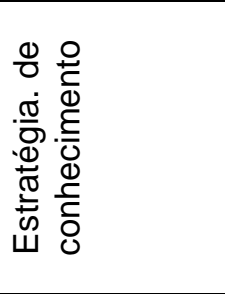 } & $\begin{array}{l}\text { Mapeamento das } \\
\text { competências }\end{array}$ & $\begin{array}{l}\text { Kaplan e Norton, } \\
\text { 2006, p. } 127\end{array}$ & Operacional \\
\hline & & $\begin{array}{l}\text { Requisitos de capacidade } \\
\text { futura }\end{array}$ & $\begin{array}{l}\text { Kaplan e Norton, } \\
\text { 2006, p. } 127\end{array}$ & Atividades \\
\hline & & Decisão de fornecimento & Lepak e Snell, 1999 & Operacional \\
\hline & \multirow{5}{*}{ 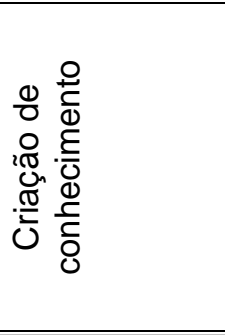 } & Desgaste criativo & $\begin{array}{l}\text { Leonard e Sensiper, } \\
1998 \\
\end{array}$ & Tático \\
\hline & & Pensamento paralelo & De Bono, 1985 & Tático \\
\hline & & Modelo SECI & $\begin{array}{l}\text { Nonaka e Takeuchi, } \\
1995\end{array}$ & Tático \\
\hline & & Equipes de especialistas & $\begin{array}{l}\text { Easterby-Smith e } \\
\text { Lyles, 2005, p.169 }\end{array}$ & Operacional \\
\hline & & Aprendizagem de laço duplo & Carroll et al., 2005 & Tático \\
\hline \multicolumn{2}{|c|}{ Regae: Rev. Gest. Aval. Educ. } & Santa Maria & Pub. contínua & p. 1-17 \\
\hline
\end{tabular}




\begin{tabular}{|c|c|c|c|c|}
\hline & & Entrevista & De Long, 2004 & Operacional \\
\hline & 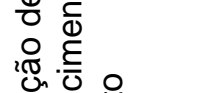 & Mapas mentais & $\begin{array}{l}\text { Gelb, 1998, p.176- } \\
177\end{array}$ & Atividades \\
\hline & $=0$ & Filmagem & De Long, 2004 & Operacional \\
\hline & $\stackrel{ \pm}{0}$ & Mentoring & De Long, 2004 & Operacional \\
\hline & & Quadro de ação estratégica & De Long, 2004 & Operacional \\
\hline & $\frac{\otimes}{2} \stackrel{0}{\frac{0}{0}}$ & $\begin{array}{l}\text { Valorização do } \\
\text { conhecimento }\end{array}$ & $\begin{array}{l}\text { Andriessen, 2004; } \\
\text { Massingham, 2014 }\end{array}$ & Atividades \\
\hline & 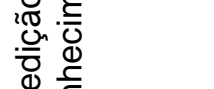 & $\begin{array}{l}\text { Métricas de mudança } \\
\text { cultural }\end{array}$ & Massingham, 2012 & Estratégico \\
\hline & $\Sigma$ & Gestão de risco & Massingham, 2010 & Estratégico \\
\hline$\stackrel{ }{\stackrel{0}{ }}$ & 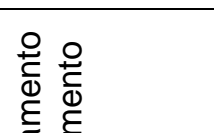 & $\begin{array}{l}\text { Barreiras de } \\
\text { compartilhamento de } \\
\text { conhecimento }\end{array}$ & Massingham, 2010 & Tático \\
\hline 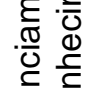 & 胥言 & Mudança cultural & $\begin{array}{l}\text { MacDermott e } \\
\text { O'Dell, } 2001\end{array}$ & Tático \\
\hline$\frac{\overline{0}}{\overline{0}} \overline{0}$ & 응 & Comunidades de prática & Gorelick et al., 2004 & Atividades \\
\hline$\circlearrowleft$ 응 & రั & Motivação & $\begin{array}{l}\text { Davenport e Probst, } \\
2002\end{array}$ & Tático \\
\hline & Objetivo & Ferramentas & Autores & $\begin{array}{l}\text { Nível } \\
\text { organizacional }\end{array}$ \\
\hline & & $\begin{array}{l}\text { Habilidades de comunicação: } \\
\text { narrativas }\end{array}$ & Bartel e Garud, 2005 & Tático \\
\hline & & $\begin{array}{l}\text { Habilidades de comunicação: } \\
\text { narração de histórias }\end{array}$ & Denning, 2005 & Tático \\
\hline & & $\begin{array}{l}\text { Habilidades de comunicação: } \\
\text { conversação }\end{array}$ & Ipê, 2007 & Tático \\
\hline & & $\begin{array}{l}\text { Habilidades de comunicação: } \\
\text { escuta }\end{array}$ & Abrashoff, 2002 & Tático \\
\hline & & $\begin{array}{l}\text { Habilidades de comunicação: } \\
\text { apreciação }\end{array}$ & Abrashoff, 2002 & Tático \\
\hline & & $\begin{array}{l}\text { Habilidades de comunicação: } \\
\text { entender a lacuna entre o } \\
\text { conhecedor e o buscador }\end{array}$ & Massingham, 2010 & Tático \\
\hline & & Conhecer o gap & $\begin{array}{l}\text { Pfeffer e Sutton, } \\
2000\end{array}$ & Operacional \\
\hline & $\frac{\Phi}{0} \cdot \frac{5}{0}$ & Auxílio de pares & Gorelick et al., 2004 & Atividades \\
\hline & $\stackrel{D}{D} \frac{\Phi}{C}=$ & Revisões após ações & Senge et al., 2005 & Atividades \\
\hline & वे & Retrospecto & $\begin{array}{l}\text { Collison e Parcell, } \\
2004\end{array}$ & Tático \\
\hline & & $\begin{array}{l}\text { Modelo de contabilidade de } \\
\mathrm{RH}\end{array}$ & Lepak e Snell, 1999 & Operacional \\
\hline & 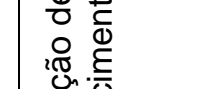 & Barreiras de aquisição & $\begin{array}{l}\text { Holsapple e Joshi, } \\
2000\end{array}$ & Operacional \\
\hline & 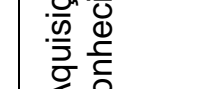 & Eficiência de mercado & $\begin{array}{l}\text { Huizing e Bouman, } \\
2002\end{array}$ & Tático \\
\hline & & Especificação do contrato & Aadne et al., 1996 & Tático \\
\hline & & Corretores de conhecimento & Cillo, 2005 & Tático \\
\hline & & Selecionar processo & Probst et al., 2002 & Tático \\
\hline & & Armazenamento & Srikantaiah, 2007 & Atividades \\
\hline & $\Phi 0 \subseteq \frac{1}{0}$ & Metadados & Inmon et al., 2007 & Atividades \\
\hline & 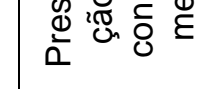 & $\begin{array}{l}\text { Banco de dados de lições } \\
\text { aprendidas }\end{array}$ & $\begin{array}{l}\text { Hubert e O'Dell, } \\
2007\end{array}$ & Atividades \\
\hline
\end{tabular}

Fonte: Adaptada de Massingham (2014). 
Para Massingham (2014), no grupo de gestão de recursos do conhecimento ocorrem as decisões em termos de planejamento, gerenciamento de riscos e orçamentos. Nesse grupo, encontram-se as ferramentas relacionadas à estratégia do conhecimento, à criação do conhecimento, à retenção do conhecimento e à medição do conhecimento. Todas induzem à gestão do conhecimento mais pontualmente.

No segundo grande grupo de ferramentas o objetivo é o de gerenciar o fluxo e os gatilhos para o conhecimento. De acordo com Massingham (2014), no fluxo, encontramse o compartilhamento, o uso, a aquisição e a preservação do conhecimento, promovendo, assim, os sistemas que movem o conhecimento nas organizações, os quais dão fluidez tanto internamente quanto para o conhecimento externo da organização.

As ferramentas tecnológicas formam outro grupo de ferramentas necessárias à gestão do conhecimento nas organizações, devidamente explicitadas no quadro 3 . Dividem-se em duas características: organização/armazenamento do conhecimento e geração e distribuição e acúmulo de conhecimento. Além disso, também são analisadas à luz das características culturais que favorecem a sua utilização.

Quadro 3 -

Ferramentas tecnológicas que atuam na gestão do conhecimento e características ligadas à cultura organizacional para sua implantação.

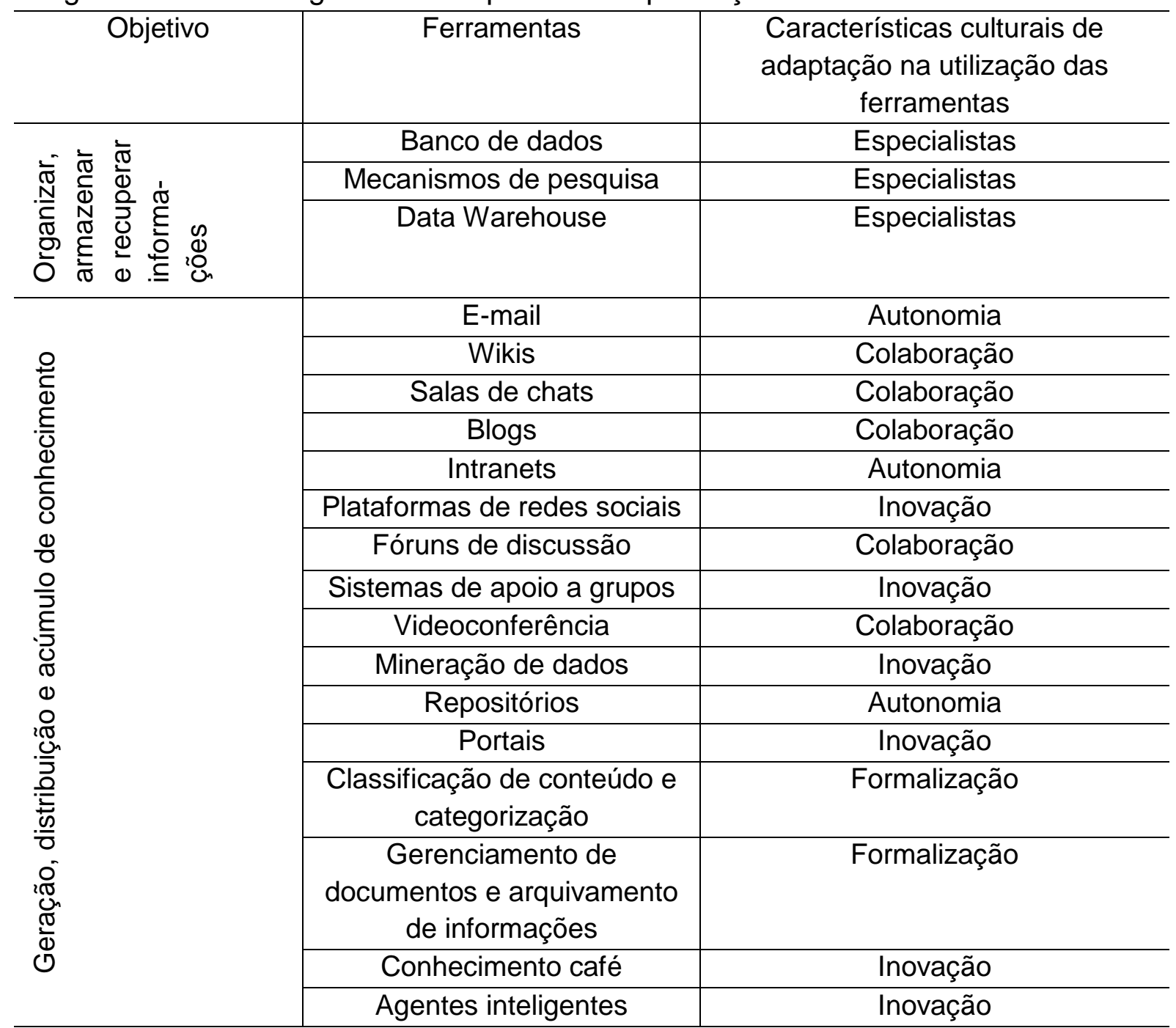

Fonte: Adaptada de Alavi, Kayworth e Leidner (2005). 
Em conformidade com os referidos autores, se compatíveis às características culturais da organização, os objetivos das ferramentas tecnológicas podem ser desenvolvidos com mais eficácia. Tais características são as expostas na última coluna do quadro 3 e mostram que os objetivos podem ser alcançados com maior facilidade diante da implantação de ferramentas adequadas às diferentes culturas.

\section{Procedimentos metodológicos}

O objeto de estudo da pesquisa foi a gestão do conhecimento numa IES privada do Estado do Paraná, de sede única e mantida por pessoa jurídica, de direito privado, cuja finalidade é educacional, científica e cultural. A IES desenvolve suas atividades desde 0 ano de 1998 e exemplifica uma classe de pequenas IES que buscam sua permanência no mercado por meio de diferenciação estratégica e vantagem competitiva sustentada.

A pesquisa descritiva realizada, de caráter teórico-empírico seguiu o método de estudo de caso, sob a abordagem quantitativa e qualitativa, como caráter intervencionista (Gil, 2008). Nessa perspectiva, o método indutivo foi utilizado para a formulação de um projeto de gestão do conhecimento específico e o meio utilizado para investigação foi um instrumento de pesquisa - questionário, adaptado da ideia original de Bukowitz e Williams (2002).

O referido questionário, validado para aplicação em IES, apresentou resultados satisfatórios quanto à consistência interna. Das 112 perguntas que o estruturam, foram retiradas 28 com menor correlação após análise de confiabilidade aplicada em IES.

O respectivo instrumento de pesquisa compõe-se de sete partes e determina os pontos fortes e fracos do processo de gestão do conhecimento na instituição. As perguntas contêm três opções de respostas e se classificam em nível baixo, nível médio e nível alto de concordância. Para cada resposta há uma multiplicação de pontos: para fraco, multiplica-se por um; para médio, por dois; para forte, por três. O resultado é dividido por 48. A soma das sete seções dá o valor máximo de 336, conforme adaptação feita no próprio instrumento. Com esse cálculo, obtém-se a percentagem relativa a cada seção, e o diagnóstico torna-se estruturado e de interpretação setorizada.

A amostragem para este estudo foi não probabilística, por acessibilidade, pois, conforme determinam Marconi e Lakatos (2000), não é possível a aplicação de quaisquer fórmulas estatísticas por cálculo de amostra, devendo, portanto, os elementos serem selecionados pela facilidade de acesso.

A amostra da pesquisa originária deste estudo compõe-se de 18 questionários, os quais foram enviados eletronicamente a gestores acadêmicos e administrativos, bem como a professores da IES que se apresentam como unidades de observação. Do total encaminhado, 14 questionários foram respondidos, representando $77,77 \%$ de retorno.

$\mathrm{Na}$ pesquisa também se buscou um amplo levantamento das ferramentas de implantação da gestão do conhecimento na literatura nacional e internacional. Por conseguinte, foram utilizadas bases de dados dos periódicos da Coordenação de Aperfeiçoamento de Pessoal de Nível Superior com os termos de busca em artigos: ferramentas de gestão do conhecimento, OR tools, Knowledge Management em títulos, resumos ou palavras-chave, em dois idiomas: português e inglês. 
O resultado da busca correspondeu a 1.097 artigos. Na sequência, acrescentou-se a estes o filtro para NOT system e NOT sistemas, resultando em 572 artigos. Ainda, os resultados foram refinados com o tópico Knowledge Management, contemplando 36 artigos. Após a leitura dos resumos, foram excluídos 27 artigos por serem incompatíveis à pesquisa de ferramentas de gestão do conhecimento, resultando num total de 09 artigos condizentes à utilização de ferramentas e práticas de gestão do conhecimento.

\section{Análise dos resultados}

O perfil dos sujeitos respondentes compõe-se de $50 \%$ de professores e $50 \%$ gestores que ocupam funções administrativas. Os resultados dos questionários são apresentados na tabela 1 e demonstram o nível de desenvolvimento de cada seção. Consoante Bukowitz e Williams (2002), as pontuações para organizações que aplicaram empiricamente o diagnóstico de gestão do conhecimento ficaram em média de 30 a $70 \%$ para cada seção.

Tabela 1 -

Resultados da pesquisa.

\begin{tabular}{l|c|c}
\hline Seção & Total da etapa ${ }^{*}$ & Percentual da etapa (\%) $^{* *}$ \\
\hline Processo tático & & 72,60 \\
\hline Obtenha & 488 & 71,41 \\
\hline Utilize & 480 & 73,35 \\
\hline Aprenda & 493 & 71,12 \\
\hline Contribua & 478 & \\
\hline Processo estratégico & & 58,47 \\
\hline Avalie & 393 & 69,33 \\
\hline Construa/mantenha & 466 & 68,29 \\
\hline Descarte & 459 & \\
\hline
\end{tabular}

Fonte: Elaborada pelas autoras (2019).

Notas: * valores representam o somatório de todos os questionários; ** valores representam a média do percentual de todos os questionários.

Percebe-se que, no primeiro grupo, correspondente ao processo tático, todas as quatro dimensões ficaram acima de $70 \%$. Nessa divisão o processo tático é voltado às atividades internas da instituição.

Segundo os dados do modelo utilizado acima de $70 \%$ dos resultados são satisfatórios. Logo, a gestão do conhecimento em processos internos se enquadra em um nível satisfatório. Os dados mostram uma aproximação entre as percentagens, demostrando um alinhamento forte na busca da obtenção do conhecimento, da utilização deste, do cultivo e da arte de aprender fazendo, como também da contribuição por intermédio do compartilhamento e cooperação para encontrar pontos de benefícios mútuos.

No segundo grupo, equivalente ao processo estratégico, conforme o modelo em evidência, há uma combinação entre o conhecimento interno e as exigências estratégicas com o mercado. Dessa forma, nesse grupo a atenção se volta ao conhecimento externo à IES. Em se tratando dos resultados da pesquisa, os percentuais apresentados são levemente menores em todas as seções. A primeira seção desse grupo apresenta menor 
resultado, isto é, $58,47 \%$. Esta seção é direcionada à identificação das novas formas de capital intelectual da organização, buscando avaliar o conhecimento por intermédio de índices e comunicá-los aos stakeholders. Como enfatizam Bukowitz e Williams (2002), o desafio, nessa seção, é o de visualizar as estruturas que guiam as práticas de gestão do conhecimento, bem como identificar os novos usos de conhecimento que geram capital intelectual e mensurá-los.

Nas outras duas seções, construa/sustente e descarte, o percentual mostra-se um pouco abaixo do desejado, uma vez que, segundo o modelo, é de $70 \%$. Ou seja, respectivamente, correspondem a $69,33 \%$ e $68,29 \%$. Para as autoras, na seção construa/sustente, o desafio é o de alinhar a tecnologia de informação às pessoas, bem como utilizar o conhecimento para fortalecer o relacionamento com o cliente. Já na seção descarte, o desafio é reconhecer a necessidade de deixar conhecimentos que, um dia, foram úteis, mas se tornaram obsoletos, identificando as formas de conhecimento que podem ser alavancadas e as que são limitadas, além de distinguir e dispensar os verdadeiros drenos de recursos.

Com base nos dados analisados, infere-se que os resultados apresentam algumas deficiências no processo estratégico da gestão do conhecimento da IES, tendo em vista o ambiente externo. O menor índice encontra-se na seção avalie que põe em evidência a avaliação do conhecimento por índices, direcionados a todos os stakeholders da Instituição.

Na sequência, as seções construção e manutenção do conhecimento externo, assim como descarte do conhecimento são as que apresentam deficiências que devem ser atendidas pelo projeto de gestão do conhecimento ora proposto, composto por ferramentas escolhidas especificamente para atender a essas necessidades.

Para Rathi e Given (2017), as ferramentas dependem de diversos fatores como tamanho da organização, infraestrutura tecnológica, características do segmento e questões culturais. Por isso, a proposição do projeto de gestão do conhecimento fundamenta-se no alinhamento das características ambientais da IES pesquisada, em sua cultura organizacional e nos valores intrínsecos às necessidades não supridas por um método informal de gestão do conhecimento, haja vista que a instituição em questão não contém uma gestão do conhecimento estruturada.

Por intermédio do questionário aplicado, pôde-se perceber uma característica cultural da organização que se volta à colaboração e à inovação. Por essa razão, o primeiro grupo de ferramentas propostas tem, inerentemente, características voltadas à externalização, pois foi uma das necessidades apontadas nos resultados. Destaca-se que esse primeiro grupo compõe-se de ferramentas tecnológicas.

Distintamente, o segundo grupo de ferramentas propostas busca suprir a lacuna demonstrada pelos resultados no processo estratégico do conhecimento e se relaciona à avaliação e à divulgação do mesmo, bem como ao descarte de conhecimentos que não são considerados mais necessários. Esse grupo apresenta caráter não tecnológico. 
Quadro 4 -

Proposição de projeto para estruturação da gestão do conhecimento.

\begin{tabular}{|c|c|c|c|c|}
\hline \multirow{4}{*}{ 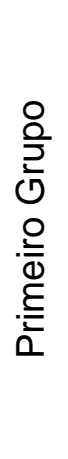 } & $\begin{array}{l}\text { Caract. ambiental } \\
\text { para adaptação da } \\
\text { ferramenta }\end{array}$ & Ferramenta & Finalidade & $\begin{array}{l}\text { Nível organiza- } \\
\text { cional em que } \\
\text { opera }\end{array}$ \\
\hline & Colaboração & Wikis & $\begin{array}{l}\text { Armazenar } \\
\text { conhecimento }\end{array}$ & Operacional \\
\hline & Colaboração & Blogs & $\begin{array}{l}\text { Difundir } \\
\text { conhecimento }\end{array}$ & Tático \\
\hline & Inovação & Portais & $\begin{array}{l}\text { Externalizar } \\
\text { conhecimento }\end{array}$ & Estratégico \\
\hline \multirow{5}{*}{ 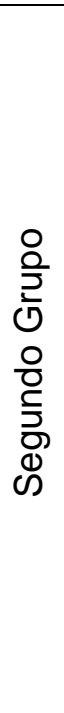 } & Inovação & $\begin{array}{l}\text { Requisitos de } \\
\text { capacidade futura- } \\
\text { Kaplan e Norton, 2006, } \\
\text { p.12 }\end{array}$ & $\begin{array}{l}\text { Estratégia de } \\
\text { conhecimento }\end{array}$ & Atividades \\
\hline & Inovação & $\begin{array}{l}\text { Quadro de ação } \\
\text { estratégica- De Long, } \\
2004\end{array}$ & $\begin{array}{l}\text { Retenção do } \\
\text { conhecimento }\end{array}$ & Operacional \\
\hline & Inovação & $\begin{array}{l}\text { Métricas de mudança } \\
\text { cultural- Massingham, } \\
2013\end{array}$ & $\begin{array}{l}\text { Medição do } \\
\text { conhecimento }\end{array}$ & Estratégico \\
\hline & Colaboração & $\begin{array}{l}\text { Gestão de risco- } \\
\text { Massingham, } 2010\end{array}$ & $\begin{array}{l}\text { Medição do } \\
\text { conhecimento }\end{array}$ & Tático \\
\hline & Inovação & $\begin{array}{l}\text { Selecionar processo- } \\
\text { Probst et al, } 2002\end{array}$ & $\begin{array}{l}\text { Preservação do } \\
\text { conhecimento }\end{array}$ & Tático \\
\hline
\end{tabular}

Fonte: Elaborada pelas autoras (2019)

A proposta desse projeto de gestão do conhecimento para a IES corresponde à atuação no armazenamento, na difusão, na retenção, na medição e na preservação do conhecimento. Todos esses aspectos sob o enfoque estratégico do mesmo. Nesse sentido, o intento é a busca pelo alinhamento das ferramentas de gestão de conhecimento às características culturais da organização para que se possa atingir um melhor desempenho nos resultados desejados.

No quadro 4 confere-se a proposição do projeto, dividido em ferramentas tecnológicas e não tecnológicas. Ressalta-se que as ferramentas tecnológicas, que compõem o primeiro grupo, têm em vista o fornecimento de conhecimentos ao público externo, compartilhando, assim, os recursos internos com os stakeholders que circundam a Instituição. Já no segundo grupo, as ferramentas não tecnológicas propostas visam ao aprimoramento da gestão interna do conhecimento, assim delineadas:

a) ferramenta "requisitos de capacidade futura": fornece conhecimento futuro, utilizando-se de princípios do Balanced Scorecard;

b) ferramenta "quadro de ação estratégica": retém o capital intelectual da organização já existente tanto em relação aos funcionários quanto às estruturas existentes; 
c) ferramenta "métricas de mudança cultural": mede as percepções das pessoas em relação à cultura organizacional e acrescenta mecanismo nas métricas de mudanças cultural relacionadas à gestão do conhecimento;

d) ferramenta "gestão de risco": identifica os riscos associados aos recursos de conhecimento e aloca uma pontuação de risco, adaptada de uma ferramenta clássica de gerenciamento de risco;

e) ferramenta "seleção de processo": identifica o conhecimento a ser preservado, assim como fornece estrutura para que este conhecimento seja capturado e atualizado constantemente.

Com a devida distribuição de propósitos na combinação de ferramentas tecnológicas e não tecnológicas, o intento foi a criação de estrutura do projeto de gestão do conhecimento para a IES analisada, fornecendo respostas às necessidades momentâneas desta, sem deixar de alinhá-las à cultura organizacional.

\section{Considerações finais}

Este estudo teve como objetivo a elaboração de um projeto de gestão do conhecimento a partir das características ambientais de gestão do conhecimento em uma IES privada do estado do Paraná. Este objetivo possibilitou investigação, descrição e análise de aspectos relevantes à pesquisa original, tais como diagnóstico da atual realidade da gestão do conhecimento em uma IES por meio das sete seções definidas por Bukowitz e Williams (2002), identificação e reconhecimento das ferramentas de gestão do conhecimento mais abrangentes na literatura acadêmica atual, além do estabelecimento de inter-relação entre as características mais frágeis elencadas após o diagnóstico da IES com as soluções propostas pelas ferramentas de gestão do conhecimento.

Para a elaboração do projeto de gestão do conhecimento, contou-se com os resultados obtidos no diagnóstico realizado empiricamente na IES, os quais apontaram as maiores necessidades na divisão de processos estratégicos do conhecimento, voltados ao ambiente externo da organização.

As ferramentas de gestão do conhecimento, oriundas dos resultados da revisão bibliográfica, viabilizaram o apontamento de características que as classificam como tecnológicas e não tecnológicas. As tecnológicas se dividem em grupos com determinadas especificidades, isto é, organização/armazenamento de conhecimento, bem como geração, distribuição e acúmulo do mesmo.

Diferentemente, nas ferramentas de gestão do conhecimento não tecnológicas, os grupos divisores se classificam como gestão de recursos de conhecimento - estratégia, criação, retenção e medição do conhecimento - e gerenciamento do conhecimento compartilhamento, uso, aquisição e preservação do conhecimento. Enfatiza-se que a inter-relação ocorreu por intermédio da adequação das necessidades da IES e características das ferramentas, resultando num projeto de gestão do conhecimento à IES.

Sendo assim, o projeto proposto compôs-se de ferramentas tecnológicas: wikis, blogs e portais, e também não tecnológicas: requisito de capacidades futuras, quadro de ação estratégica, métricas de mudança cultural, gestão de risco e seleção de projetos. 
Ao concluir este estudo, depreende-se que, mesmo com algumas limitações relativas ao não aprofundamento das características de aplicabilidade de cada ferramenta selecionada no projeto, há uma significativa contribuição não somente à IES pesquisada, mas a outras instituições que desejam estruturar sua gestão do conhecimento.

Isso abre possibilidades para que tais características de aplicabilidade sejam estudadas empiricamente em cada uma das ferramentas explicitadas em pesquisas futuras.

\section{Referências}

ALAVI, Maryam; KAYWORTH, Timothy; LEIDNER, Dorothy. An empirical examination of the influence of organizational culture on knowledge management practices. Journal of management information systems, v. 22, n. 3, 2005, p. 191-224. Disponível em: <https://www.jmis-web.org>. Acesso em: 23 jun. 2018.

BARNEY, Jay. Organizational culture: can it be a source of sustained competitive advantage? Academy of management review, California, v. 11, n. 3, 1986, p. 656-665.

BUKOWITZ, Wendi. R; WILLIAMS, Ruth. L. Manual de gestão do conhecimento: ferramentas e técnicas que criam valor para a empresa. Porto Alegre: Bookman, 2002.

CALDAS, Sólon Hormidas. Gestão do conhecimento em IES: um estudo de caso. Brasília: UCB, 2009. 113f. Dissertação (mestrado em Informática). Universidade Católica de Brasília.

CURADO, Carla; BONTIS, Nick. Parallels in knowledge cycles. Computers in Human Behavior, v. 27, n. 4, 2011, p. 1438-1444. Disponível em: <https://dl.acm.org/citation.cfm?id=J1034\&picked=prox>. Acesso em: 12 jun. 2018.

DAVENPORT, Thomas. H. Conhecimento empresarial. Rio de Janeiro: Elsevier, 1998.

DAVENPORT, Thomas. H; PRUSAK, Laurence. Working knowledge: how organizations manage what they know. Harvard Business Press, 1998.

DEMSETZ, Harold. The nature of the firm revisited. Oliver E. Williamson and Sidney G. Winter, eds, 1991. New York, Oxford University Press.

DONATE, Mario Javier; GUADAMILLAS, Fátima. Organizational factors to support knowledge management and innovation. Journal of Knowledge Management, v. 15, n. 6, p. 890-914, 2011. Disponível em: <https://www.emeraldinsight.com/journal/jkm>. Acesso em: 5 jul. 2018.

EDVINSSON, Leif; MALONE, Michael. S. Intellectual capital: realizing your company's true value by finding its hidden brainpower. New York: Harper Business, 1997.

FERENHOF, Helio Aisenberg; BIALECKI, Mariana Zaniboni; DURST, Susanne; SELIG, Paulo. Análise das dimensões do capital intelectual: uma revisão da literatura. In: VAZ, Caroline Rodrigues (org.). Capital intelectual: reflexão da teoria e prática. Florianópolis: UFSC, 2014, p. 20-49.

FIGUEIREDO, Saulo Porfírio. Gestão do conhecimento: estratégias competitivas para a criação e mobilização do conhecimento na empresa. Rio de Janeiro: Qualitymark, 2005.

GIL, Antonio Carlos. Métodos e técnicas de pesquisa social. São Paulo: Atlas, 2008.

GRANT, Robert M. Toward a knowledge-based theory of the firm. Strategic Management Journal, v. 17, n. S2, p. 109-122, 1996. 
KIANTO, Ainó; RITALA, Paavo; SPENDER, John. C; VANHALA, Mika. The interaction of intellectual capital assets and knowledge management practices in organizational value creation. Journal of Intellectual Capital. v. 15, n. 3, 2014, p. 362-375. Disponível em: <https://www.emeraldinsight.com/journal/jic>. Acesso em: 6 jun. 2018.

KRÜCKEN-PEREIRA, Lia; COSTA, Marilia Damiani; BOLZAN, Ariovaldo. Gestão do conhecimento aplicada ao desenvolvimento de novos produtos. Revista Inteligência Empresarial, v.12, p. 48-56, 2002.

MARCONI, Marina; LAKATOS, Eva. Maria. Metodologia científica: ciência e conhecimento científico, métodos científicos, teoria, hipóteses e variáveis, metodologia jurídica. São Paulo: Atlas, 2000.

MASSINGHAM, Peter. An evaluation of knowledge management tools: part 1-managing knowledge resources. Journal of Knowledge Management, v. 18, n. 6, 2014, p. 10751100. Disponível em: <https://www.emeraldinsight.com/journal/jkm>. Acesso em: 5 jul. 2018.

NONAKA, Ikujiro; TAKEUCHI, Hirotaka. The knowledge-creating company: how Japanese companies create the dynamics of innovation. New York: Oxford University Press, 1995.

NONAKA, Ijujiro; TAKEUCHI, Hirotaka. Criação do conhecimento na empresa: como as empresas japonesas geram a dinâmica da inovação. Rio de Janeiro: Elsevier, 2004.

RATHI, Dinesh; GIVEN, Lisa. M. Non-profit organizations' use of tools and technologies for knowledge management: a comparative study. Journal of Knowledge Management, v. 21, n. 4, 2017, p. 718-740. Disponível em: <https://www.emeraldinsight.com/journal/jkm>. Acesso em: 6 jul. 2018.

SPENDER, J. C. Making knowledge the basis of a dynamic theory of the firm. Strategic Management Journal, v. 17, n. 2, 1996, p. 45-62.

STEWART, Thomas. A. A riqueza do conhecimento: o capital intelectual e a organização do século XXI. Rio de Janeiro: Campus, 2002.

TERRA, José Cláudio Cyrineu. Gestão do conhecimento: o grande desafio empresarial. Rio de Janeiro: Negócio, 2000.

WENGER, Etienne; MCDERMOTT, Richard Arnold; SNYDER, William. Cultivating communities of practice: a guide to managing knowledge. Boston: Harvard Business Press, 2002.

Simone Boruck Klein é estudante no Programa de Mestrado Profissional da Fundação Pedro Leopoldo.

Orcid: https://orcid.org/0000-0003-4956-7182.

Endereço: Rua Pedro Ivo, 2376 - 85813230 - Cascavel - PR - Brasil.

E-mail: simoneboruck@uol.com.br.

Salette Silveira Azevedo é estudante no Programa de Mestrado Profissional da Fundação Pedro Leopoldo.

Orcid: https://orcid.org/0000-0002-5099-9938.

Endereço: Rua Primeiro de Maio, 554/501 - 83323-020 - Pinhais - PR - Brasil.

E-mail: salettesilveiraazevedo@gmail.com. 
Paula Bitar Silva é estudante no Programa de Mestrado Profissional da Fundação Pedro Leopoldo.

Orcid: https://orcid.org/0000-0003-4531-7450.

Endereço: Rua 60, 74/401 - 35179-000 - Santana do Paraíso - MG - Brasil.

E-mail: paulabitar@yahoo.com.br.

Recebido em 28 de março de 2019.

Aceito em 9 de maio de 2019.

(c) (i) 